\title{
A Schematic Overview of Addiction: Molecular Effects of Cocaine, Methamphetamine and Morphine on Limbic Neurons
}

\author{
Ümit Sayın H*
}

Turkey

ISSN: 2578-0042

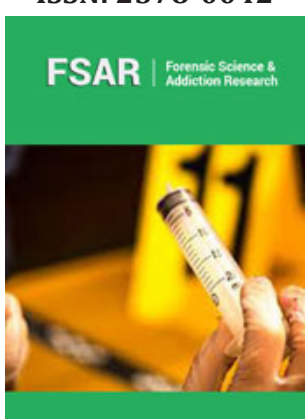

*Corresponding author: Ümit Sayın H, Turkey

Submission: 睯 March 29, 2019

Published: 㭗April 30, 2019

Volume 4 - Issue 4

How to cite this article: Ümit Sayın $H^{*}$, A Schematic Overview of Addiction: Molecular Effects of Cocaine, Methamphetamine and Morphine on Limbic Neurons. Forensic Sci Add Res. 4(4). FSAR.000599.2019.

DOI: 10.31031/FSAR.2019.04.000599

Copyright@ Ümit Sayın H, This article is distributed under the terms of the Creative Commons Attribution 4.0 International License, which permits unrestricted use and redistribution provided that the original author and source are credited.

\begin{abstract}
Abuse of methamphetamine, cocaine and morphine is an increasing global problem. According to 2018 UNODOC report between 2012 and 2016, 151 millions of people used cannabis; 146 millions of people consumed cocaine; 275 millions of people used opioids and opiates; 131 millions of people consumed amphetamines. According to another Global Drug Survey-2017, the figures of addiction may be much worse than the expected. People use cocaine and amphetamines because these drugs increase the dopamine release in ventral tegmental area (VTA), nucleus accumbens (NA) and prefrontal cortex, brain regions which take part in the reward-pleasure circuitry. They also play major roles in the development of addiction. In the review the mechanism of action of amphetamines, cocaine and morphine are explained in a very clean schematic way; explaining why people get addicted to these drugs. Cocaine binds strongly to the dopamine-reuptake transporter, preventing the reuptake of dopamine into the nerve terminal Because of this blocking effect, dopamine remains at high concentrations in the synapse and continues to affect adjacent neurons, producing the characteristic cocaine "high".
\end{abstract}

Activated D1 receptor activates the PKA signaling pathway, and this pathway plays a critical role in mediating the behavioral responses to cocaine administration. Cocaine-induced neuroadaptations, including dopamine depletion, may underlie craving and hedonic dysregulation. Acute administration of amphetamine induces phosphorylation of cAMP response element-binding protein (CREB) and expression of several immediate early genes (IEGs), such as c-fos. The IEGs is likely to initiate downstream molecular events, which may have important roles in the induction and maintenance of addictive states. Chronic exposure to amphetamine induces a unique transcription factor delta FosB, which plays an essential role in long-term adaptive changes in the brain. Morphine can cause indirect excitation of VTA dopamine neurons by reducing inhibitory synaptic transmission mediated by GABAergic neurons. The chronic use of morphine is characterized by adaptive changes in neurons and neuronal communication; such adaptations (e.g., 'super-activation' of adenylyl cyclase) must underlie altered behavior associated with morphine dependence and withdrawal syndrome, as well as drug-induced craving and relapse to drug use.

Keywords: Addiction; Cocaine abuse; Methamphetamine abuse; Opiate abuse; Morphine; Ventral tegmental are; VTA; Nucleus accumbens; NA; CREB; c-fos; Dopamine; D1 receptor; NMDA-R

\section{Introduction}

Illegal drug consumption is a serious growing global problem today. Most of these psychoactive drugs do not only give extreme harms to the health of people, but also creates huge criminal problems which ruin many families and lives as well as inducing other social catastrophes. Illegalizing drugs and policing on drug users did not work during the last century. United Nations and other agencies are seeking for alternative solutions to combat against the global drug problem, such as legalizing and thus controlling the drug usage moderately. According to UNODOC-2018 Report, between 2012 and 2016, 151 million of people used cannabis; 146 million of people consumed cocaine; 275 million of people used opioids and opiates; 131 million of people consumed amphetamines [1]. Between 2006 and 2017, Opioid usage dropped significantly, while the consumption of other synthetic drugs, cannabis and stimulants increased immensely. The global drug trade is never ceasing, and it seems that it will never come to an end. Policing methods are creating more criminal, legal and social problems. We are mentioning about an annual global market of 500 billion to 900 billion dollars at least. Drug cartels are easily buying many corrupted government officials and law enforcement agencies. Under these circumstances enforcement and policing the social systems are only serving the drug Lords and cartels. 
Another 2017-academic study among 115000 people revealed that the actual numbers are far more serious than the UNUDOC report as seen in the below plot [2]; (Figure1). If among randomly chosen correspondents and subjects $(\mathrm{N}=115000)$, a total number of $57.3 \%$ use stimulants, $92.4 \%$ consume hallucinogens, and $9.7 \%$ use narcotic analgesics (of course there may be multiusers, using many of them at the same time), then this means that Global Drug War is LOST and there should be other precautions to be taken!

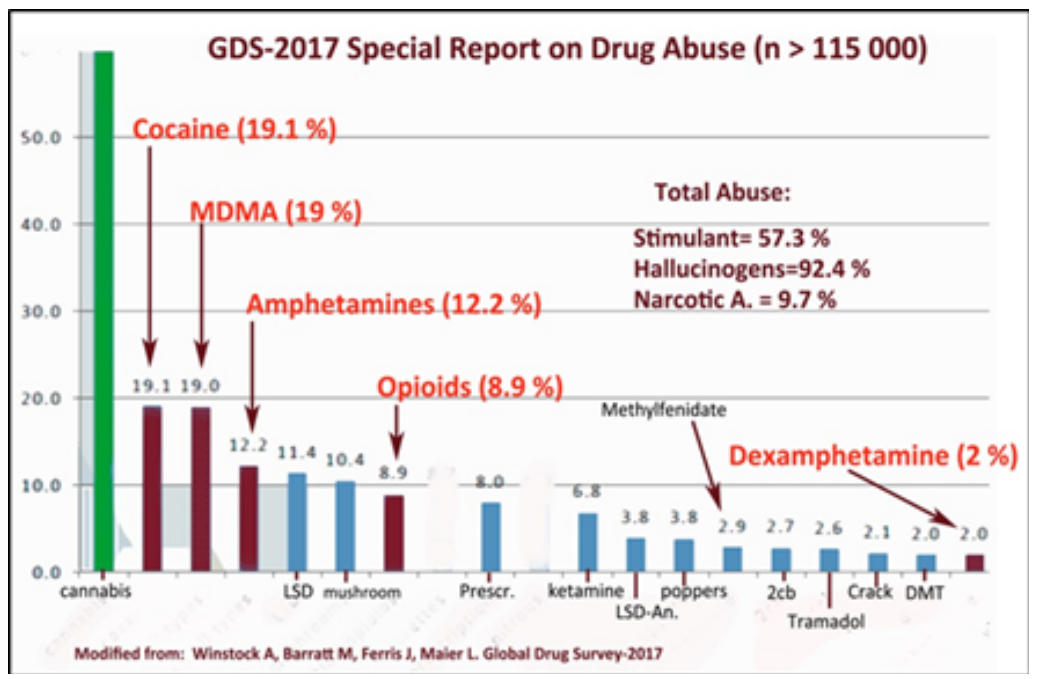

Figure 1: GDS-2017 report shows that the figures of addiction may be much worse than the UN-reports.

To create alternative methods, we have to face a couple of facts after asking a series of questions. the first question is; "Why such a majority of people use drugs?". The following questions will be:

1. Do these drugs harm human health and nervous system?

2. If yes. What are the methods we should proceed with to prevent this hazardous effect son human health?

3. What can be the possible effects of these drugs on human health? Are these effects irreversible and persistent?

4. How should we treat drug addiction and other health problems induced along with the drug abuse?

5. Do the policing methods work at all?

6. Can we prevent current widespread drug use?
7. If answer to Q7 is NO. Will legalization of drugs work to prevent or at least decrease drug related problems?

Although there are numerous investigations, animal model studies, clinical studies, human PET and fMRI investigations, we are not still at the point of determining the unified perspective to decide about how these drugs influence human health and nervous system; how hazardous they are; how tenacious the neurophysiological changes; and how these alterations can be coped with and treated. There are some theories and hypothesis about the mechanism of how addiction, craving and abstinence syndromes develop. There are some proposed treatment methods which are partially healing abstinence or craving. This review article will deal with the first and second questions above (Figure 2A).
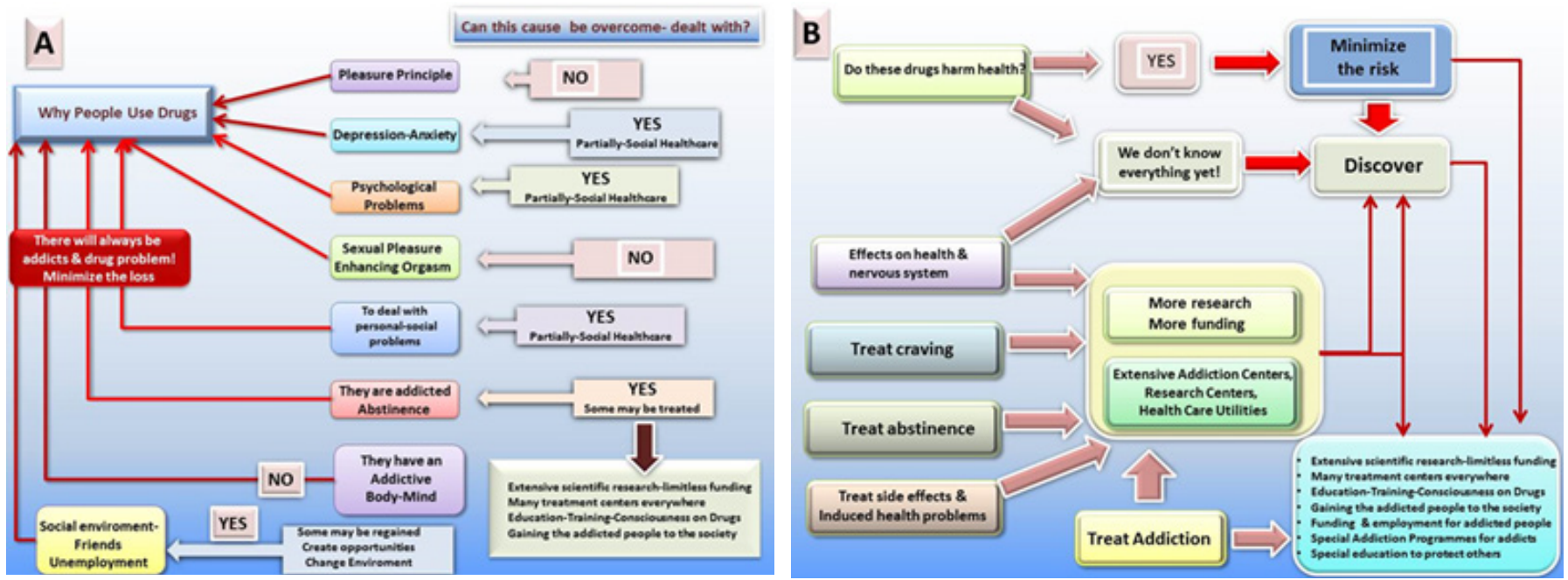

Figure 2A \& 2B: Steps to be taken to prevent addiction and treat addicted people 
Some people, who are genetically more prone, get addicted to drugs very easily; some do not! Some stimulant drugs have effects of euphoria, increased libido, enhanced sexual pleasure and orgasm due to the sudden rush of dopamine and norepinephrine. Most people like those rewarding effects. The effect of those addicting drugs are more effective than anti-depressants (e.g. SSRIs) or anxiolytics (e.g. benzodiazepines). So, there is no potential way of preventing drug abuse totally, today. The health agencies and institutions should focus on decreasing the hazards and health problems of drug addiction, as well as treating addiction, after discovering the mechanisms of addiction. For this limitless research funds should be directed to academic and scientific research to find out the mechanisms of craving, abstinence and addiction. Also, very extensive addiction centers should be funded using today' scientific information, as well as education-training programmed for the public should be started all over the world (Figure 2B).

Current policing methods have not worked for the last 150 years; on the contrary, global drug problem have become much worse. The acceleration of global drug problem is increasing every year and becoming exponential every decade. The drug addicts are not the ones who should be blamed, but the corrupted governments that do not see obvious facts and indirectly support or unconsciously facilitate the growth of Cartels and Drug Mafiosa.

\section{Why Do People Use Cocaine and Amphetamines?}

Humans are hedonist and pleasure discovering-seeking higher primates [3-5]. Contentment, pleasure, liking, wanting, sexual pleasure and satisfaction, orgasm, are all positive emotions that lead to the general states of mind, "contentment and "happiness"; without those pleasurable effects, life would be less meaningful. Being happy is a combination of various effective components. Central nervous system structures are such organized and specialized that there are some pleasure regions, pleasure-reward circuitry which passes neurological signals by means of some modulatory neurotransmitters, such as in, glutamate, dopamine, GABA, endogenous opioids, oxytocin and norepinephrine etc. Pleasure principal uses biological processes which are inherited and have descended from our grand ancestors, coded in our DNAs. These processes include, making the animals and/or mammals and higher primates become habituated to pleasure inducing events or "rewards". People, all their lives, run, strive, crave and race for various kind of rewards: reward of "feeling good; good taste and smell; satisfying their ego; sexual pleasure and orgasm; chocolate; junk food; buying as they wish; possessing; making money, getting richer and more powerful etc.

The animal brain and reward mechanisms are not different, but simpler. It is easier to construct experiments and establish experimental models in animals, since we cannot use invasive techniques in higher primates and humans. Evolution shows us that we have very similar built-in mechanisms and circuits in the brain for a certain mind states, such as depression, fear, anxiety and pleasure. For instance it was possible to design medication to treat epilepsy, after discovering some of the mechanisms of epileptic discharges and using anti-epileptic agents on animals, which is a valid, working and pragmatic method today. In this century, most of the epileptic cases can be treated. A drug, such as sodium valproate, vigabatrin (GVG) or benzodiazepines which block electroshock, pentylenetetrazole (PTZ) or kindling seizures in animal models, will also abolish seizures in humans [6]. Pleasure, reward and addiction studies were also made with the same notion; actually the pleasure and reward centers were first discovered in animals.

A reward activates the reward-pleasure system or hedonic hotspots and generates feelings of favorable emotions (e.g., liking). At the same time, a reward also activates motivational systems either originating from the limbic system or the anatomical pleasure regions and produces incentive behavior (e.g., wanting). For example, when rats press a key and receive electrical stimulation through an electrode implanted in the hypothalamus, they try to press the key continuously as they can to get more electrical stimulations, which is the well documented "brain stimulation reward (BSR)."

The electrical stimulation of some subcortical structures, such as the nucleus accumbens (NA), the lateral hypothalamus, and the ventral tegmental area (VTA), have been proven to induce strong behaviors that are associated with motivations in animals. It should always be kept in mind that "wanting" and "liking" are two different feelings. "Wanting" is caused by the innate and built in approach because of the fragrance, taste, or outer appearance of the object (such as food), it is a response of the organism towards an incentive salience. In liking there is the "pleasure" factor, and it is a learned behavior, involving short term memory. The dopaminergic, oxytocinergic and glutamatergic systems (D1-like dopamine and NMDA receptors) play important roles in the occurrence of and learning of pleasure [7-9]

The results coming from animal studies (mostly rats), have been tested in live human subjects by using very sophisticated imaging techniques, such as fMRI. It was shown that, when there is pleasure the following areas of the brain may become activated, like experimental animals [8,10-15]:

1. Thalamus and/or lateral hypothalamus

2. Nucleus accumbens (NA)

3. Ventral tegmental area (VTA)

4. Ventral pallidum

5. Insula

6. Cingulate cortex

7. Prefrontal cortex

8. Orbitofrontal cortex

9. Septum

Humans chase pleasure and contentness; he/she escapes from dysphoria, stress, anxiety and depression and choses the behaviors that lead him/her to euphoria, anxiolysis, relieving his/ her fears, dysphoria and depression. For instance, drug usage, alcohol addiction, over eating etc. are the clinical signs of the 
attempts to relieve anxiety and depression and attain euphoria, mild sedation, elevated mood and attain contentment. Central nervous system (CNS) stimulants, such as cocaine, amphetamine, methamphetamine increase the neurotransmitter concentrations by various mechanisms; once more neurotransmitters, such as dopamine and norepinephrine, stay longer in the synaptic cleft in certain areas, they increase the formation of action potentials, and increase the synaptic transmission. When it comes to sexual pleasure, particularly for women, orgasmic experience gives antidepressant, anxiolytic, sedative and analgesic effects [3-5;1621] mostly because of the rush of the following neurochemicals $[3,15,19,22,23]$.
1. Dopamine
2. Oxytocin
3. Prolactin
4. Epinephrine \& norepinephrine

\section{Endorphins}

People use cocaine, amphetamines, MDMA, methamphetamine or C-Meth because they have very acute effects and they have very positive effects on the mood, such as:

1. Euphoria, contentment and happiness feelings

2. Anti-depressant effects

3. Anxiolytic effects

4. Increased motivation and attachment to life

5. Pleasurable effects

6. Increasing libido, sexual pleasure and enhancing orgasm

7. Making the person more alert and wakeful

8. Increasing the mental capacities, IQ memory and recollection (Figure 3).

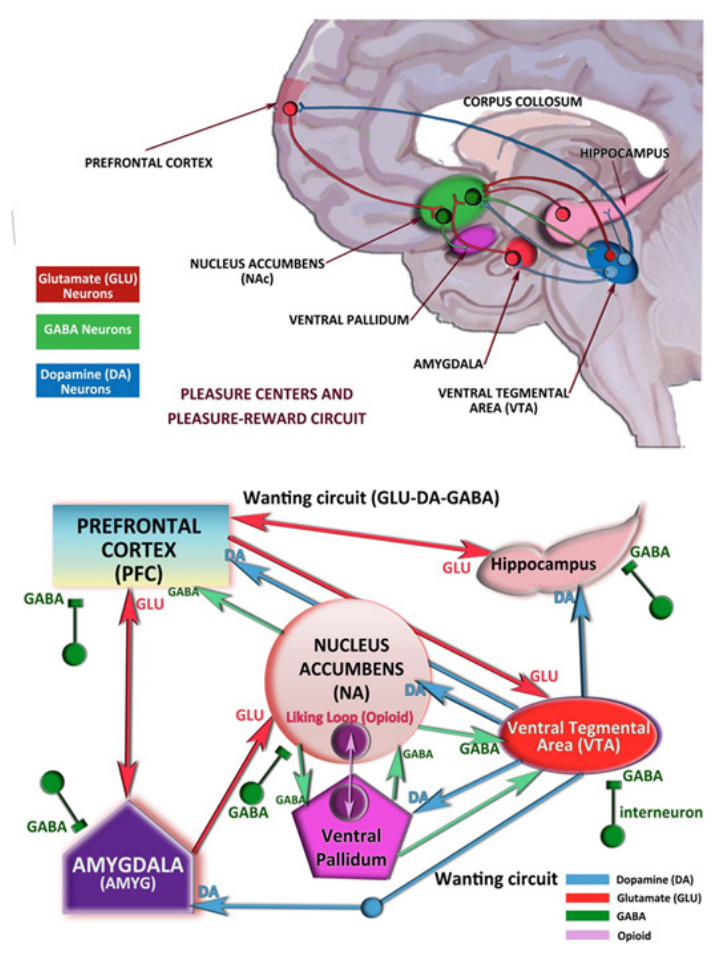

Figure 3: The pleasure centers and pleasure-reward circuitry in the limbic system.

\section{Dopamine}

Neuropharmacological studies show that dopamine is the main neurotransmitter, which mediates pleasure reaction, while some dopamine agonists and antagonists (e.g. into lateral hypothalamus or VTA) modulated the pleasure and BSR. It is shown that the medial forebrain bundle includes massive dopamine fibers. Both the mesocortical dopamine fibers and the nigrostriatal dopamine fibers pass through the lateral hypothalamus, so that the stimulation of lateral hypothalamus seems to induce excessive release of dopamine. Dopamine (DA) is an excitatory or inhibitory neurotransmitter in the central nervous system. DA receptors have subtypes which are mainly two families D1-like (D1, D5) and D2-like (D2, D3, D4) groups. Activation of D1- group receptors increases cyclic adenosine 3, 5, -monophosphate (cAMP) through stimulation of adenylyl cyclase via $G_{s}$ stimulatory G-proteins (Figure $4 A$ ). But activation of D2group receptors decreases cAMP through $\mathrm{G}_{\mathrm{i}}$ (inhibitory) pathway. The other receptor subtypes belong to the D2-like subfamily (D2, D3, and D4) and they are $\mathrm{G}_{\mathrm{i}}$ protein-coupled receptors that inhibit adenylyl cyclase and activate $\mathrm{K}^{+}$channels [24,25].

The cAMP is formed from the ATP through an enzyme adenylyl cyclase (AC) and it is metabolized by the enzyme phosphodiesterase (PDE) which exists in the cytoplasm. Drugs or agents (such as 
caffeine) that block phosphodiesterase (PDE) activity, also block the inhibition. In the central nervous system, the inhibition of inhibition also results in excitation. When the cAMP concentrations rise, cAMP-dependant protein kinase (PKA), phosphorylates receptors and channels, and activates important transcription factors like cyclic adenosine monophosphate response-element binding protein (CREB) [26]. cAMP is a second messenger responsible for the cellular effects of multiple hormones and neurotransmitters via activation of its main effector, protein kinase A (PKA) (Figure 4B).
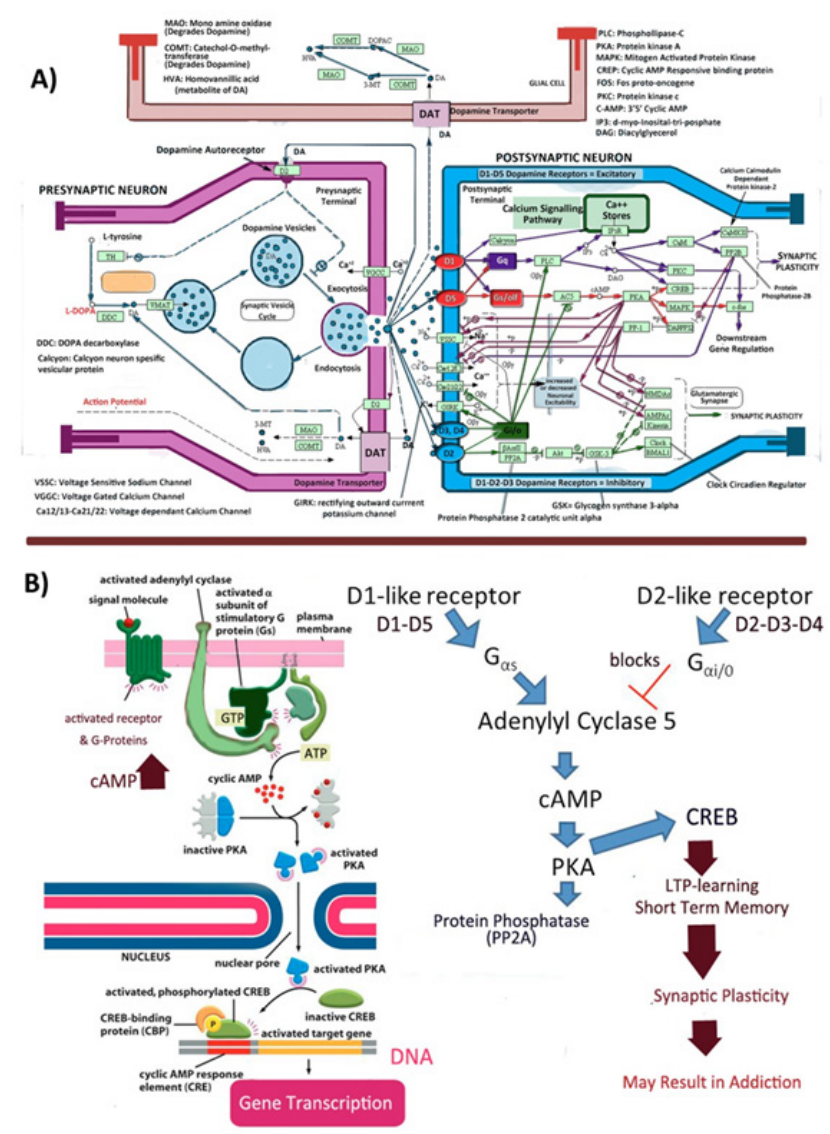

Figure 4A: Dopamine synapse in the limbic system.

Figure 4B: Dopamine receptors and intracellular molecular events when dopamine binds to D1-like or D1-like DA receptors.

Endogenous DA levels and DA release increase in the Nucleus Accumbens (NA), Ventral Tegmental Area (VTA) and Orbitofrontal Cortex (ORB-C) and thalamus (THL) when the CNS stimulant drugs are used such as nicotine, cocaine and methamphetamine [27] and also these stimulant substances are associated with the reward system in those areas by means of increasing the release of dopamine extremely, compared to the usual-daily reward system. Reward system of DA in the brain includes the VTA and NA, where a sequence of reactions occurs based on the drug stimuli and behavior, such as drug seeking and eventually the consumption of that preferred drug.

These drugs stimulate mainly every part of the brain, but particularly VTA, prefrontal cortex and NA as well as some other subcortical structures of the limbic system. Extracellular DA release induces action potential firing at D1 dopaminergic receptors and in some people they may start and extreme craving [28]. In people who continuously use CNS stimulants, this craving is correlated with the concentration of DA released into the synaptic cleft [29].
Craving is an electro-chemical gained behavior hypothesized to be establishing new adaptation of the mesolimbic dopaminergic system [8]. In animal and human studies self-administration abuse of drugs that elevate dopamine levels, creates some positiveseeming clinical effects, such as pleasure, euphoria, increased libido, and enhanced sexual pleasure, cessation of anxiety, hedonism, and loss of the depressive mood.

Cellular mechanisms in addiction also involve glutamatergic receptors and glutamate (GLU). In pleasure and hedonism mechanisms AMPA, NMDA and metabotropic glutamate receptors also play important roles. As well known, NMDA receptors are responsible for the short term learning mechanisms and long term potentiation (LTP), a form of electrophysiological learning and conditioning. These two receptor systems and the neurotransmitters DA and GLU are widely distributed in the brain, particularly prefrontal cortex, limbic system, basal ganglia (NA, VTA, Putamen etc.) which play important roles in emotions, motivation, learning, memory, sexual behavior and orgasm. Most of the pleasurable 
acts, sexual activity or orgasmic climaxes are associated with dopamine release in many parts of the brain. Actually, many other neurotransmitters or hormones are released during high pleasure and climax states, such as oxytocin, norepinephrine, prolactin and endogenous opiates $[30,31]$.

It is assumed that cross molecular signaling of dopaminergic and combined molecular signaling of dopaminergic and glutamatergic systems, particularly through dopamine (D-1) and glutamate receptors (NMDA and AMPA) play critical roles in the cellular transcriptional chains which affect the synaptic plasticity and learning, by means of reorganizing the neural networks initially, which eventually results in the alteration of emotions and behavior, as well as psychological responses of the individual $[30,31]$.

\section{Mechanism of Action of Methamphetamine and Cocaine} on Synaptic Transmission

Both cocaine and amphetamines (particularly methamphetamine) seem to influence human mood very fast and in a positive way in the beginning. Actually they are powerful mood modulators and anti-depressants when taken through intranasal (cocaine) or oral (methamphetamine) routes; crystal methamphetamine (C-meth) may also be inhaled after it is heated

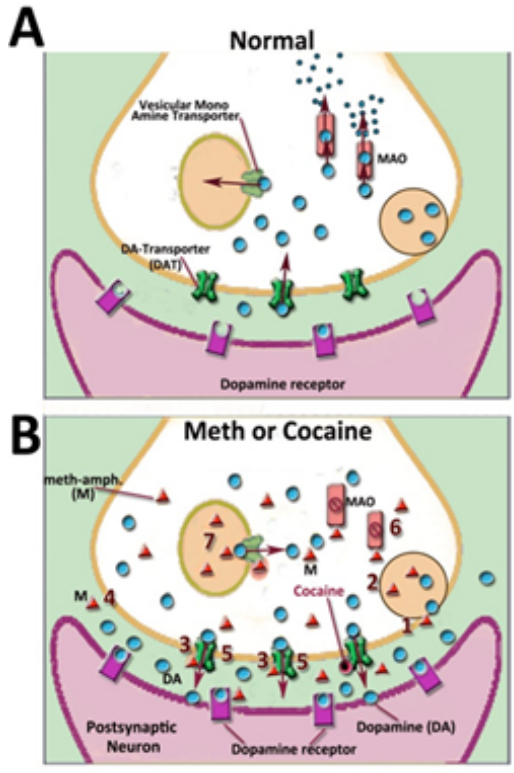

into gaseous form, while C-meth's evaporation heat is $212{ }^{\circ} \mathrm{C}$. Cocaine has an abrupt mood changing effect lasting for 30 to 40 minutes, while C-meth's effects last for 24-30 hours when taken orally and a couple of hours when inhaled. Both of them increase libido and makes sexual pleasure and orgasms more intense both in men and women. The hypothesis that they create physiological addiction is debatable, but they induce powerful psychological addiction [32,33].

Cocaine mainly binds to Dopamine (DA) transporter at the presynaptic membrane and blocks the re-uptake of dopamine molecules to be degraded by MAO or COMT; thus, increases DA concentration and the probability of it to bind postsynaptic DAreceptors. DA stays longer in the synaptic cleft. However, this effect lasts for 30-40 minutes if new cocaine doses are not inhaled again. The euphoria effect of cocaine is higher than amphetamines and C-meth.

Amphetamines, Methamphetamine or C-meth has a more profound and long-lasting effect, because it increases the DA concentrations in a couple of ways, while also stimulating the DA release into the cleft. Amphetamines and C-Meth do not only increase DA but also norepinephrine (NE) as well. Methamphetamines realize this action in a couple of different ways (Figure 5):

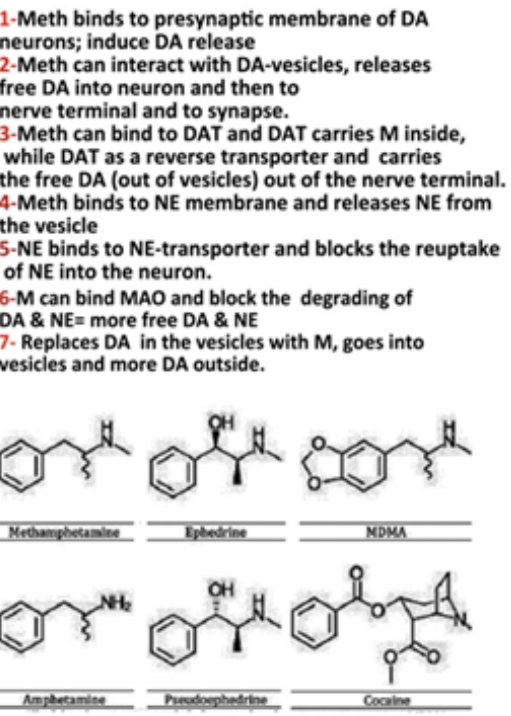

Figure 5: The mechanism of action of methamphetamine and cocaine.

1. Meth binds to the presynaptic membrane and induces the release of DA into the cleft, it's a specific effect directly on the DA vesicles.

2. Meth can interact with the free DA in the presynaptic neuron and releases DA either from the vesicles and also induces free DA into the cleft.

3. Meth binds to NE and DA transporter and DAT or NEtransporter carries Meth into the cytoplasm, while it reverse transports DA and NE into the extracellular synaptic cleft.
4. Meth blocks the re-uptake of DA and NE into the neuron, thus increases the concentration of DA and NE in the synaptic cleft.

5. At higher concentrations, Meth enters the neuron, and invade the synaptic vesicles of DA or NE, thus forcing and replacing a lot of DA and NE molecules out of the vesicles. Thus, free DA and NE which are out of the vesicles increase. And they are reverse transported by DAT or NET into the cleft. Meanwhile Meth makes itself to be carried into the neuron binding to DAT and NET, such that more meth is inside the cell and it induces free DA and NE molecules to be reverse transported outside. 
6. Meth binds to MAO and blocks the degrading of DA and NE, so it increases the duration of existence of these monoamines.

7. Meth may also interact the monoamine vesicle transporter, either blocking it, or making itself to be carried into vesicles such that free DA or NE molecules stay in the cytoplasm much longer while some of them are carried outside either by diffusion, exocytosis or active reverse transport by DAT and NET [32,33].

The acute effects of cocaine are as follows:

1. It increases the feeling of well-being and contentment.

2. Increased euphoria.

3. Increased libido

4. Increased sexual pleasure

5. Enhanced and powerful orgasms.

6. Antidepressant effects.

7. Mood elevation

8. Increased alertness and alert consciousness

1. The acute effects of methamphetamines are as follows:

2. At low doses: (oral 10-30mg)

1. All of the cocaine's effect

2. Increased self esteem

3. Very powerful wakefulness

4. Increased IQ and EQ

5. Increased empathy

6. Powerful Anxiolytic \& Anti-depressant effects.

7. Increased higher mental functions

8. Increased memory and learning

9. Increased associations

10. Increased creativity

11. Loss of sleep

12. Very increased libido

13. Powerful sexual pleasure

14. Enhanced and prolonged orgasms (particularly for women, high doses of Meth may induce erection problems in men since it stimulates the adrenergic system; systemic beta blocker my alleviate this problem)

15. Increased mental and emotional capacities.
3. At high doses (oral 50-75mg)

1. All of the above low dose effects

2. Increase of the all mentioned mental capacities.
3. Paranoia may elevate in some people; paranoid thinking.

4. Mild or acute visual hallucinations.

5. May precipitate psychosis and/or schizophrenia in propsychotic people

6. Severe delusions and persecution delusions

7. Uncontrolled thoughts.

8. Some sexual paraphilias may emerge

9. Unmasking of sub consciousness.

\section{Mechanisms of Action of Morphine and Opioid Agonists} on Synaptic Transmission

All four opioid receptors contain seven-transmembrane proteins that couple to inhibitory G proteins. When they are activated by an agonist, such as endorphin, or exogenous agonists, such as morphine, the $G \alpha$ and $G \beta \gamma$ subunits dissociate [34,35]. Former opioid receptor pharmacology has proven that guanosine triphosphate (GTP) modulates agonist binding to opioid receptors (OR). It was afterwards established that GTPase activity is stimulated by opioid agonists. Today it is generally known that all four known OR types couple to pertussis-toxin-sensitive G proteins [36]. The most crucial aspect of OR signal transduction shows that opioids modulate calcium and potassium ion channels. After $\mathrm{G} \alpha$-i dissociated from $\mathrm{G} \beta \gamma$, the $\mathrm{G} \alpha$ protein subunit moves to interact with the G-protein gated inwardly rectifying potassium channel, Kir3. Channel deactivation happens. This process causes cellular hyperpolarization and inhibits the formation of successive action potentials and decreases neural activity. In many studies the inhibitory effects of OR on neural excitability were shown to be formed by interactions of opioid receptors with $G$ protein-regulated inwardly rectifying potassium channel (Kir3) [37-41].

When they are activated, all of the OR causes a reduction in $\mathrm{Ca}^{+2}$ currents which are sensitive to P/Q-type, $\mathrm{N}$-type, and L-type channel blockers. OR-induced inhibition of calcium currents is mediated by binding of the dissociated G $\beta \gamma$ subunit directly to the membrane receptor channel. This binding decreases the voltage activation of channel and hence decreases the probability of the pore opening. Many studies have shown that opioid receptors interact $\mathrm{Ca}^{+2}$ channels and induce configurational alterations; if the agoniststimulated opioid receptor is postsynaptic, it will take the resting membrane potential to the reversal potential of potassium, which is around $-90 \mathrm{mV}$; thus allowing the cell become more hyperpolarized. If the opioid receptor is presynaptic, then it will block any kind of synaptic neurotransmission or neurotransmitter release; since without inward calcium currents the neurotransmitter vesicles will not activate and fuse with the presynaptic membrane. It was also shown that MOR Stimulation results in G protein-dependent inhibition of $\alpha-1 \mathrm{~A}$ and $\alpha-1 \mathrm{~B}$ subunits [42-45].

It is well known that the acute administration of opioid agonists reduces $\mathrm{Ca}^{+2}$ dependent release of synaptic vesicles; this event can be regarded as one of the factors that is responsible of 
opiate tolerance $[46,47]$. In addition, because the activation of $\mu, \gamma$ and $\kappa$ opioid receptors inhibits adenylyl cyclase activity, the AMPdependent $\mathrm{Ca}^{+2}$ influx is also reduced [48]. As a summary when an agonist binds to $\mu$-opioid receptor (MOR) the mechanism of action of MOR receptor is seen as:

1. Inhibition of Adenylyl cyclase (AC)and the formation of cAMP is decreased. This makes the cell less excitable.

2. Galpha-i/o subunit affects the AC activity which is inhibited. This decreases cAMP. Then cAMP induced PKA (protein kinase A) activity is decreased. This results in the reduced opening of voltage gated calcium channels (VGCC); less calcium enters during voltage drops of the membrane. This induces hyperpolarization.

3. Activation of PKC (Protein kinase C) and CREB gene transcription may result in many other membrane proteins to be synthesized which may induce up regulation or downregulation of opioid receptors or related other receptors.

4. Galpha-i/o working as an inhibitory electrophysiological factor stimulates the G-protein activated inwardly rectifying potassium channels (GIRK). More potassium currents make the membrane less excitable and hyperpolarized.

5. As a result, the activity of many voltage gated calcium channels are decreased (including L, M, N types), near to this hyperpolarizing potassium channels are opened and inward rectifying potassium currents makes the membrane hyperpolarized and preventing an action potential to fire.

\section{Cocaine addiction}

Cocaine addiction is a chronic, recurring behavioral disorder in which compulsive drug-seeking and drug-consuming behavior is predominant. There is data that the dopaminergic system that projects from the ventral tegmental area (VTA) into the nucleus accumbens (NAc). Cocaine interacts with the dopamine-reuptake transporter, and it prevents the re-uptake of dopamine into the glial cells or presynaptic neuron to be recycled. Thus DA stays at higher concentrations in the synaptic cleft and stimulates the D1 receptors at neighboring neurons or the post synaptic membrane. Activating D1 receptors stimulate PKA signaling which results in many chemical reactions and cascade of events which may lead to synaptic plasticity. [49,50] in Figure 5-7.

\section{Methamphetamine addiction}

Methamphetamine is a stimulant of CNS which induces severe addiction. Addictive stimulant drugs increase extracellular concentrations of DA in NA and PFC, which are accepted as projection areas of mesocorticolimbic DA neurons and important parts of the "brain reward circuit". Acute administration of methamphetamine induces phosphorylation of cAMP response element-binding protein (CREB) and expression of a number of immediate early genes (IEGs), such as c-fos. The IEGs is likely to initiate many molecular events, which may have important roles in the formation of addictive behavior which is also a learned phenomenon. Chronic exposure to methamphetamine induces the transcription factor delta FosB, which plays an essential role in the development of LTP and synaptic plasticity [51-57] in Figure 5-7.

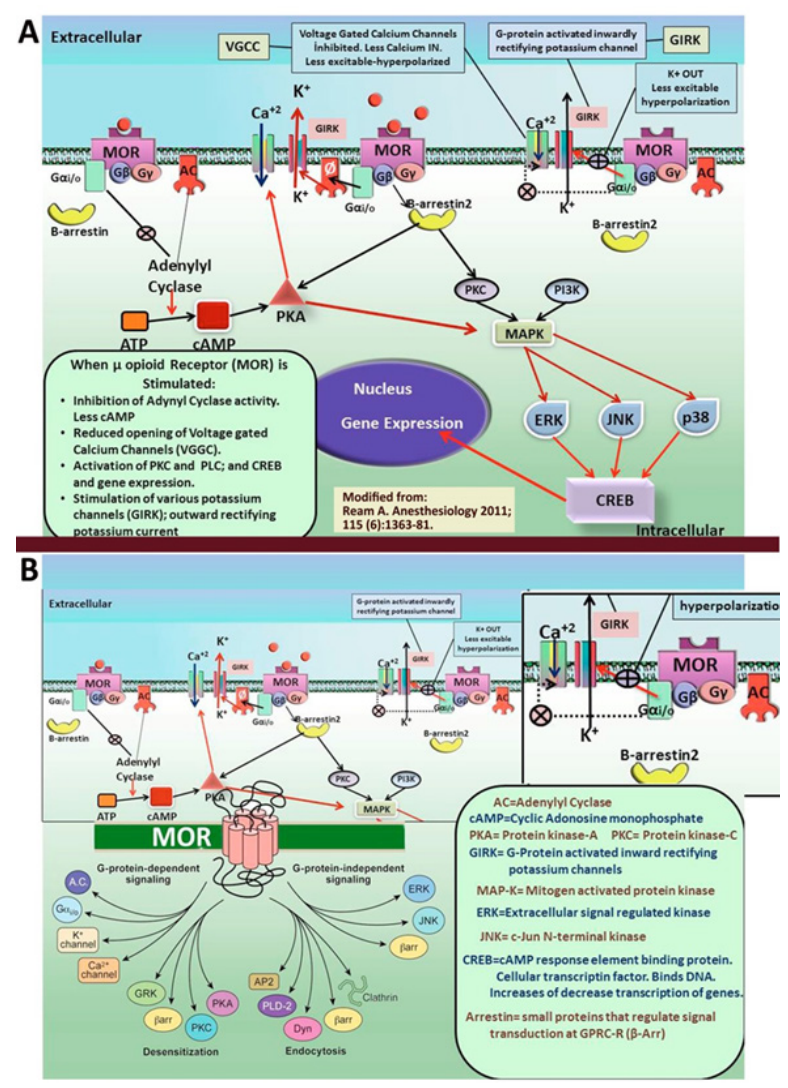

Figure 6: The mechanism of action of opioid receptors. 

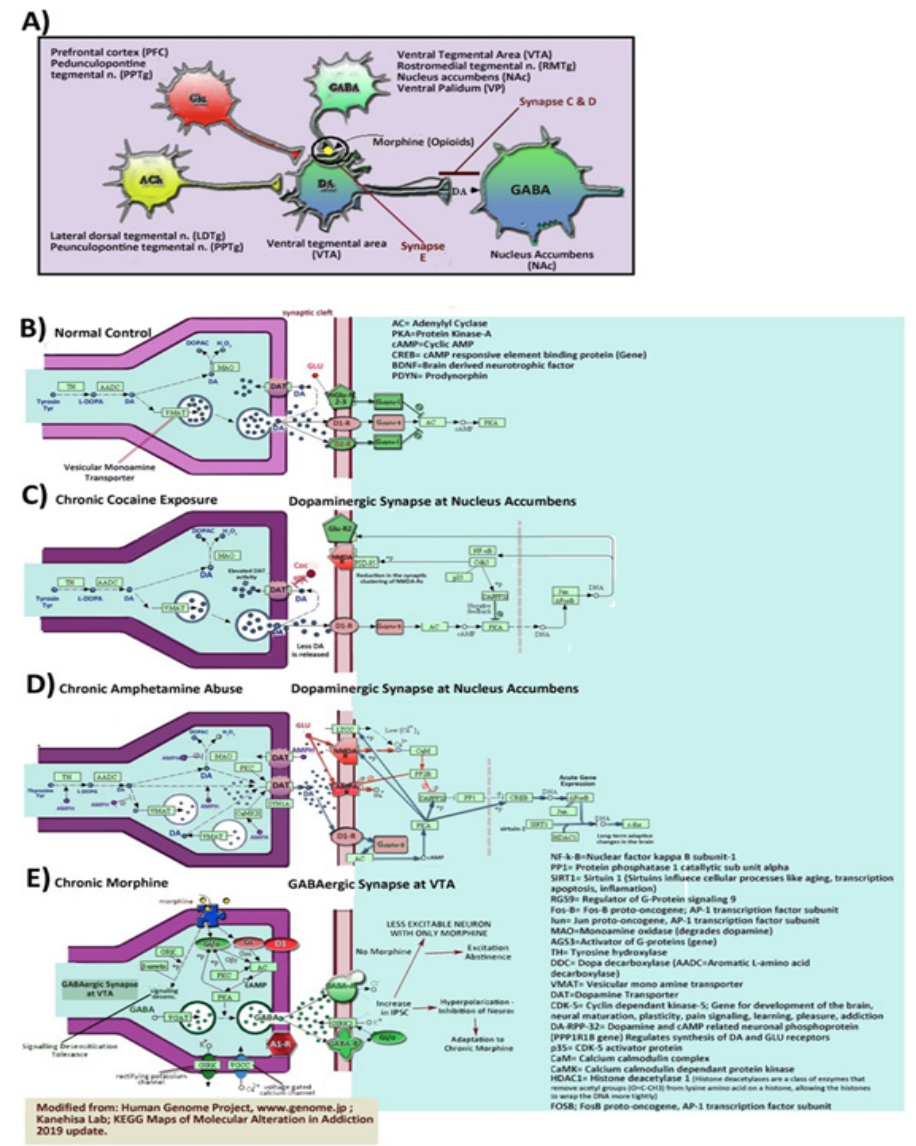

Figure 7: The schematic summary of the molecular effects of chronic methamphetamine, cocaine and morphine consumption and abuse.

\section{Conclusion}

After using animal models of reward-pleasure and addiction experiments we learned a lot from the animal models about the mechanism of action of cocaine, amphetamines and morphine, after acute or chronic administration of these drugs. We know which areas of the brain are influenced and how some genes are activated which may lead to up-regulation or down-regulation of some specific receptors as well as the increased or decreased synthesis, storing and the release of these addicting drugs.

More research funds should be directed to addiction research, because today it became possible to treat, craving, abstinence and eventually addiction. However, we are at the beginning steps to solve the main problem. Also, while the global drug abuse increases, the funds and researches on drug addiction are decreasing day by day, gradually. Science and scientists may say the last words to treat craving for drugs and also prevent abstinence syndrome, while eventually treating addiction. More research on the molecular effects of chronic abuse of these drugs should be made and more funds should be saved for science, scientists and scientific research on addiction.

\section{References}

1. (2018) United Nations UNUDC Report, in five volumes.

2. Adam W, Monica B, Jason F, Larissa M (2017) Prepared by the GDS Core Research Team. Global Drug Survey, London, England, UK.
3. Sayin HÜ (2017) Women and orgasm: Orgasm methods and new sexual therapy theories, Onur Publications, Tantra Academy, İstanbul, Turkey.

4. Sayin HÜ (2017-b) Tantra, ESR and the limits of female potentials (Review). Sexus Journal 2(3): 055-074.

5. Sayin HÜ (2017-c) Female orgasmic consciousness: new horizons (Review). Sexus Journal 2 (4): 117-145.

6. Sayın HÜ (2017-d) A bioelectrical Tornado in the hippocampus: mechanisms of temporal lobe epilepsy. Neuro Quantology 15: (1): 92103.

7. Berridge KC, Robinson TE, Wayne JA (2009) Dissecting components of reward: liking, wanting and learning. Curr Opin Pharmacol 9(1): 65-73.

8. Berridge KC, Kringelbach ML (2015) Pleasure systems in the brain. Neuron 86(3): 646-664.

9. Koob GF, Simon EJ (2009) The neurobiology of addiction: Where we have been and where are we going? J Drug Issues 39(1): 115-132.

10. Komisaruk BR, Beyer-Flores C, Beverly W (2006) The science of orgasm, John Hopkins University Press, Baltimore, USA.

11. Jannini EA, Wise N, Frangos E, Komisaruk BR (2018) Peripheral and central neural bases of orgasm. In: Goldstein WS, et al. (Eds.), Texbook of Sexual Function and Dysfunction: Diagnosis and Treatment, ( $1^{\text {st }}$ edn), John Wiley \& Sons Ltd, New York, USA, Chapter 13, pp: 179-195.

12. Berridge KC, Aldridge JW (2008) Decision utility, incentive salience, and cue-triggered wanting. In: Bargh J and Morsella E (Eds.), The psychology of action, Oxford University Press, New York, USA.

13. Castro DC, Berridge KC (2017) Opioid and orexin hedonic hotspots in rat orbitofrontal cortex and insula. Proc Natl Acad Sci USA 114(43): E9125-E9134. 
14. Kringelbach ML, Berridge KC (2012) The joyful mind. Sci Am 3027(2): 44-45.

15. Meston, CM, Frohlich PF (2000) The neurobiology of sexual function. Arch Gen Psychiatry 57(11): 1012-1030.

16. Sayin HÜ (2012-a) Increased sexual satisfaction: ESR; ultra orgasm in women (ESR; Ultra Orgasm in Women). Onur Publications, Tantra Academy, Istanbul, Turkey.

17. Sayin HÜ (2012-b) Doors of female orgasmic consciousness: New theories on the peak experience and mechanisms of female orgasm and expanded sexual response (Research and Review). Neuro Quantology 10(4): 692-714.

18. Sayin HÜ (2014) Different dimensions in sexuality (Derin Sex) (Different dimensions of sexuality: deep sex), second expanded edition, two volumes) Second expanded-extended updated edition, 2 volumes. Onur Publications, Tantra Academy, Istanbul, Turkey.

19. Sayin HÜ (2018-a) Sexual Brain (Sexual Brain). Onur Publications, Tantra Academy, Istanbul, Turkey.

20. Sayin HÜ (2018-b) A comparative review of psychopharmacology of hallucinogen-induced altered states of consciousness: relation to sexuality (Review). SexuS Journal 3(7): 413-450.

21. Sayin HÜ \& KocatÜrk A (2018-c) Expanded sexual response in the human female: The mechanisms of expanded orgasms in women. SexuS Journal 3(8): 533-548.

22. Carter CS (1992) Oxytocin and sexual behavior. Neurosci Biobehav Rev 16(2): 131-144.

23. Kim WK, Schenck CH, Grant JE, Yoon G, Dosa PI, et al. (2015) Neurobiology of sexual desire. SexuS Journal 1(1): 44-076.

24. Missale C, Nash SR, Robinson SW, Jaber M, Caron MG (1998) Dopamine receptors: from structure to function. Physiol Rev 78(1): 189-225.

25. Vallone D, Picetti R, Borrelli E (2000) Structure and function of dopamine receptors. Neurosci Biobehav Rev 24(1): 125-32.

26. Terwilliger RZ, Beitner-Johnson D, Sevarino KA, Crain SM, Nestler EJ (1991) A general role for adaptations in G-proteins and the cyclic AMP system in mediating the chronic actions of morphine and cocaine on neuronal function. Brain Res 548(1-2): 100-110.

27. LeFoll, B, Diaz J, Sokoloff P (2005) A single cocaine exposure increases BDNF and D3 receptor ex-pression: Implications for drug conditioning. Neuro Report 16(2): 175-178.

28. Schultz W (2006) Behavioral theories and the neurophysiology of reward. Annu Rev Psychol 57: 87-115.

29. Volkow ND, Wang GJ, Telang F, Fowler JS, Logan J, et al. (2007) Profound decreases in dopamine release in striatum in detoxified alcoholics: possible orbitofrontal involvement. J Neurosci 27(46):12700-12706.

30. Sayin HÜ and Schenck C (2019) Getting high on dopamine: Neuroscientific aspects of pleasure: Part-1: SexuS Journal 4(11).

31. Sayin HÜ and Schenck C (2019) Neuroanatomy and neurochemistry of sexual desire, pleasure, love and orgasm, Part-2. SexuS Journal 4(11).

32. Vearrier D, Greenberg MI, Miller SN, Okaneku JT, Haggerty DA (2012) Methamphetamine: History, pathophysiology, adverse health effects, current trends and hazards associated with the clandestine manufacture of methamphetamine. Dis Mon 58(2): 38-89.

33. Hyman SE (1996) Addiction to cocaine and amphetamine. Neuron 16(5): 901-904.

34. Childers SR, Snyder SH (1978) Guanine nucleotides differentiate agonist and antagonist interactions with opiate receptors. Life Sci 23(7): 759761.
35. Childers SR, Creese I, Snowman AM, Synder SH (1979) Opiate receptor binding affected differentially by opiates and opioid peptides. Eur J Pharmacol 55(1): 11-18.

36. Barchfeld CC, Medzihradsky F (1984) Receptor-mediated stimulation of brain GTPase by opiates in normal and dependent rats. Biochem Biophys Res Commun 121(2): 641-648.

37. Wickman K, Clapham DE (1995) Ion channel regulation by G proteins. Physiol Rev 75(4): 865- 85.

38. Sadja R, Alagem N, Reuveny E (2003) Gating of GIRK channels: Details of an intricate, membrane-delimited signaling complex. Neuron 39(1): 9-12.

39. Ippolito DL, Temkin PA, Rogalski SL, Chavkin C (2002) N-terminaltyrosine residues within the potassium channel Kir3 modulate GTPase activity of galphai. J Biol Chem 277(36): 32692-32696.

40. Torrecilla M, Quillinan N, Williams JT, Wickman K (2008) Pre and postsynaptic regulation of locus coeruleus neurons after chronic morphine treatment: A study of GIRK-knockout mice. Eur J Neurosci 28(3): $618-624$.

41. Torrecilla M, Marker CL, Cintora SC, Stoffel M, Williams JT, et. al. (2002) G-protein-gated potassium channels containing Kir3.2 and Kir3.3 subunits mediate the acute inhibitory effects of opioids on locus ceruleus neurons. J Neurosci 22(11): 4328-4334.

42. Rusin KI, Giovannucci DR, Stuenkel EL, Moises HC (1997) Kappa-opioid receptor activation modulates $\mathrm{Ca}^{2+}$ currents and secretion in isolated neuroendocrine nerve terminals. J Neurosci 17(17): 6565-6574.

43. Zamponi GW, Snutch TP (2002) Modulating modulation: Crosstalk between regulatory pathways of presynaptic calcium channels. Mol Interv 2(8): 476-478.

44. Zamponi GW, Snutch TP (1998) Modulation of voltage-dependent calcium channels by G proteins. Curr Opin Neurobiol 8(3):351-6.

45. Bourinet E, Soong TW, Stea A, Snutch TP (1996) Determinants of the G protein-dependent opioid modulation of neuronal calcium channels. Proc Natl Acad Sci USA 93(4): 1486-1491.

46. Díaz A, Ruíz F, Flórez J, Pazos A, Hurlé MA (1995) Regulation of dihydropyridine-sensitive Ca channels during opioid tolerance and supersensitivity in rats. J Pharmacol Exp Ther 274(3): 1538-1544.

47. Díaz A, Flórez J, Pazos A, Hurlé MA (2000) Opioid tolerance and supersensitivity induce regional changes in the autoradiographic density of dihydropyridine-sensitive calcium channels in the rat central nervous system. Pain 86(3): 227-235.

48. Al-Hasani R, Bruchas MR (2011) Molecular mechanisms of opioid receptor-dependent signaling and behavior. Anesthesiology 115(6): 1363-1381.

49. Nestler EJ (2005) Is there a common molecular pathway for addiction? Nat Neurosci 8(11): 1445-1449.

50. Mash DC, Pablo J, Ouyang Q, Hearn WL, Izenwasser S (2002) Dopamine transport function is elevated in cocaine users. J Neurochem 81(2): 292300 .

51. Hyman SE, Malenka RC, Nestler EJ (2006) Neural mechanisms of addiction: the role of reward-related learning and memory. Annu Rev Neurosci 29: 565-598.

52. Banerjee PS, Aston J, Khundakar AA, Zetterstrom TS (2009) Differential regulation of psychostimulant-induced gene expression of brain derived neurotrophic factor and the immediate-early gene Arc in the juvenile and adult brain. Eur J Neurosci 29(3): 465-476.

53. Renthal W, Carle TL, Maze I, Covington HE, Truong HT, et al. (2008) Delta FosB mediates epigenetic desensitization of the c-fos gene after chronic amphetamine exposure. J Neurosci 28(29): 7344-7349. 
54. Robison AJ, Nestler EJ (2011) Transcriptional and epigenetic mechanisms of addiction. Nat Rev Neurosci 12(11): 623-637.

55. Dudman JT, Eaton ME, Rajadhyaksha A, Macías W, Taher M, et al. (2003) Dopamine D1 receptors mediate CREB phosphorylation via phosphorylation of the NMDA receptor at Ser897-NR1. J Neurochem 87(4): 922-934.

56. Rajadhyaksha A, Barczak A, Macías W, Leveque JC, Lewis SE, et al (1999) L-Type Ca (2+) channels are essential for glutamate-mediated CREB phosphorylation and c-fos gene expression in striatal neurons. J Neurosci 19(15): 6348-6359.

57. Konradi C, Leveque JC, Hyman SE (1996) Amphetamine and dopamineinduced immediate early gene expression in striatal neurons depends on postsynaptic NMDA receptors and calcium. J Neurosci 16(13): 42314239.

58. Bailey CP, Connor M (2005) Opioids: cellular mechanisms of tolerance and physical dependence. Curr Opin Pharmacol 5(): 60-68.

59. Williams JT, Christie MJ, Manzoni O (2011) Cellular and synaptic adaptations mediating opioid dependence. Physiol Rev 81(1): 299-343.

60. Gintzler AR, Chakrabarti S (2006) Post-opioid receptor adaptations to chronic morphine; altered functionality and associations of signaling molecules. Life Sci 79(8): 717-722.

61. Bonci A, Williams JT (1996) A common mechanism mediates long-term changes in synaptic transmission after chronic cocaine and morphine. Neuron 16(3): 631-639.

62. Liu JG, Anand KJ (2001) Protein kinases modulate the cellular adaptations associated with opioid tolerance and dependence. Brain Res Brain Res Rev 38(1-2): 1-19.

63. Tso PH, Wong YH (2003) Molecular basis of opioid dependence: role of signal regulation by G-proteins. Clin Exp Pharmacol Physiol 30(5-6): 307-316.

64. Johnson SW, North RA (1992) Opioids excite dopamine neurons by hyperpolarization of local interneurons. J Neurosci 12(2): 483-488.

65. Madhavan A, Bonci A, Whistler JL (2010) Opioid-Induced GABA potentiation after chronic morphine attenuates the rewarding effects of opioids in the ventral tegmental area. J Neurosci 30(42): 14029-14035.

66. Bonci A, Williams JT (1997) Increased probability of GABA release during withdrawal from morphine. J Neurosci 17(2): 796-803. 